ISSN 0258-7122

Bangladesh J. Agril. Res. 39(3): 437-446, September 2014

\title{
LEACHING LOSSES OF NITROGEN, PHOSPHORUS AND POTASSIUM FROM THE SANDY LOAM SOIL OF OLD BRAHMAPUTRA FLOODPLAIN (AEZ-9) UNDER CONTINUOUS STANDING WATER CONDITION
}

\author{
M. N. ISLAM ${ }^{1}$, M. M. RAHMAN ${ }^{2}$, M. J. A. MIAN ${ }^{3}$ \\ M. H. KHAN ${ }^{4}$ AND R. BARUA ${ }^{5}$
}

\begin{abstract}
An experiment was conducted at the net house of the Department of Soil Science, Bangladesh Agricultural University (BAU), Mymensingh, Bangladesh during February to June (boro season) of 2009. The objective was to find out the leaching loss of N, P, and K in the Old Brahmaputra Floodplain Soil under continuous standing water (CSW) condition. The soil was sandy loam in texture having $\mathrm{pH} 6.6$, total $\mathrm{N} 0.08 \%$, available $\mathrm{P} 7.00 \mathrm{mg} / \mathrm{kg}$, exchangeable $\mathrm{K}$ $0.07 \mathrm{me} / 100 \mathrm{~g}$ soil, and available $\mathrm{S} 7.5 \mathrm{mg} / \mathrm{kg}$. The experiment was laid out in completely randomized design with three replications. There were six treatments, such as $\mathrm{T}_{0}$ (control), $\mathrm{T}_{1}\left(\mathrm{~N}_{120} \mathrm{P}_{25} \mathrm{~K}_{60} \mathrm{~S}_{20}\right.$ recommended dose), $\mathrm{T}_{2}$ $\left(\mathrm{N}_{180} \mathrm{P}_{37} \mathrm{~K}_{90} \mathrm{~S}_{30} \mathrm{~kg} / \mathrm{ha}\right.$ i.e., $150 \%$ of the recommended dose), $\mathrm{T}_{3}\left(75 \% \mathrm{~N}\right.$ of $\mathrm{T}_{1}$ from chemical fertilizer and $25 \% \mathrm{~N}$ from cowdung $2.5 \mathrm{t} / \mathrm{ha}$ and PKS of recommended dose from chemical fertilizer on the basis of PKS content in cowdung), $\mathrm{T}_{4}$ (as $\mathrm{T}_{1}$ but $\mathrm{N}_{109} \mathrm{~kg} / \mathrm{ha}$ from USG) and $\mathrm{T}_{5}$ (as $\mathrm{T}_{1}$ but $\mathrm{N}$ applied as foliar spray). The nutrients $\mathrm{P}, \mathrm{K}$, and $\mathrm{S}$ were applied as basal dose in the pots while urea was applied in three equal splits except $\mathrm{T}_{4}$ and $\mathrm{T}_{5}$. One USG per pot was placed after 7 days of transplanting in $T_{4}$. In $T_{5}$, urea was applied as foliar spray at 10 days interval. Leachates from individual pots were collected at 15 days intervals to determine the amount of loss of NPK. Results showed that leaching loss of NPK in the sandy loam soil under CSW condition varied widely due to different treatments over time. The total leaching loss of $\mathrm{N}, \mathrm{P}$, and $\mathrm{K}$ during the growing season varied from 22.23 to $91.21,0.063$ to 1.95 , and 35.22 to $42.01 \mathrm{~kg} / \mathrm{ha}$, respectively. Application of chemical fertilizer at higher rates resulted in greater loss of nutrients. Integrated approach of fertilizer management could minimize such losses to a great extent. Application of $\mathrm{N}$ in the form of USG reduced the $\mathrm{N}$ loss significantly.
\end{abstract}

Keywords: Leaching loss, plant nutrients, sandy loam soil, continuous standing water, boro rice.

\footnotetext{
${ }^{1}$ Scientific Officer, Soil Science Division, Bangladesh Rice Research Institute (BRRI), Gazipur, ${ }^{2 \&} 3$ Professor, Department of Soil Science, Bangladesh Agricultural University (BAU), Mymenshigh, ${ }^{4}$ Scientific Officer (Plant Breeding), Bangladesh Agricultural Research Institue (BARI), Gazipur, ${ }^{5}$ Scientific Officer, Adaptive Research Division, BRRI, Gazipur, Bangladesh.
} 


\section{Introduction}

There are many interrelated factors, both natural and managerial, responsible for the deterioration of soil fertility. This decline may occur through leaching, soil erosion and crop intensive cropping (Donova et al., 1998). When we apply fertilizer in soil for obtaining maximum yield, a significant amount of nutrients is lost through leaching, which might hamper the crop production and pollutes the environment. Leaching loss varied from soil to soil and the rate of loss differed from nutrient to nutrient. Generally, $\mathrm{N}$ fertilizers are completely water soluble and a significant portion is lost through leaching. In well-drained sandy soils, much of the nitrate can be lost by leaching as water moves nitrate down through the soil profile (Camberato et al., 2008). Nitrogen was lost through leaching, both as and even after application of the ammoniacal form in a reduced soil zone. The magnitude of fertilizer- $\mathrm{N}$ leaching varies depending on soil condition and the method of fertilizer application (Velu et al., 2001; Vlek et al., 1980; Xing et al., 2000). Leaching loss of $\mathrm{N}$ occurs in the form of $\mathrm{NO}_{3}{ }^{-}$and $\mathrm{NH}_{4}{ }^{+}$from rice fields and the extent of loss by $\mathrm{NO}_{3}{ }^{-} \mathrm{N}$ is more than $90 \%$. Keeping the rice field under standing water for a long time favours more leaching loss than keeping the field under saturation or alternate wetting and drying. Surface application of nitrogenous fertilizers in light textured soil because more nitrate loss. Application of $\mathrm{N}$ fertilizers at higher doses cause higher leaching loss. Soils having low organic matter status cause more leaching loss of nitrogen than soils rich with organic matter. It is estimated that upland lateritic sandy loam soils at Bhubaneswar cause 23-24\% loss of applied nitrogen. Leaching losses in rice field studied in sandy loam mixed red and black soils of Hirakud command area are accounted at $45-46 \%$ under saturated condition, whereas, under submerged condition, the losses were $80-84 \%$ (S. K. Sahu et al., 2006). Phosphorus is less mobile in soil and leaching loss is lower as compared to other nutrients. Besides, phosphate is extremely reactive and binds strongly with aluminum, iron, manganese, calcium, and other elements present in soils. Phosphorus losses vary from one event to another depending upon amount, intensity, and duration of rainfall (Sharpley, 1997). Phosphorus inputs in the form of mineral P fertilizer and/or animal manures may be a significant non-point source of pollution as a result of P leaching (Toor et al., 2004; Gurpal et al., 2004). Fahmi et al., 2012 showed that rice straw (RS) application was effective in preventing P loss from the soil at 6 weeks after planting (WAP) and decreased the $\mathrm{pH}$ of leachate at all observations time but it increased $\mathrm{Fe}^{2+}$ concentration in leachate $(0.07-0.42 \%$ $\mathrm{Fe}^{2+}$ in the soil were leached or 2-5 folds higher than without RS application). M. W. de Oliveira et al., 2002 found that in the first three weeks, the largest losses of $\mathrm{N}$ by leaching occurred, originating from soil/sugarcane remains-N. The mean of leached $\mathrm{N}$ during the experimental period of 11 months was of $4.5 \mathrm{~kg} / \mathrm{ha}$. The mean losses of $\mathrm{K}^{+}, \mathrm{Ca}^{+}$and $\mathrm{Mg}^{2+}$ were of 13,320 , and $80 \mathrm{~kg} / \mathrm{ha}$, respectively. Generally, the amount of $\mathrm{K}$ loss from the soils is quite high. The risk of $\mathrm{K}$ 
leaching loss under humid tropical conditions is very high, especially when higher rates of $\mathrm{K}$ fertilizers are applied on freely drained soils of low CEC (Pieri et al., 1986). The proper potassium fertilization management using adequate amounts of fertilizers and crop rotation can minimize $\mathrm{K}$ losses, which is important economically and environmentally. However, such studies are very scanty under different soil, water, and agro-climatic conditions of Bangladesh. The present study was, therefore, undertaken to determine the leaching loss of $\mathrm{N}$, $\mathrm{P}$, and $\mathrm{K}$ in sandy loam soil of the Old Brahmaputra Floodplain (AEZ-9) under continuous standing water (CSW) condition.

\section{Materials and Method}

Leaching loss of NPK under continuous standing water (CSW) condition was studied through an experiment at the net house of the Department of Soil Science, BAU, Mymensingh in the boro season (February to June) of 2009. Soil was collected from the river side of Brahmaputra and it was processed by hand. The soil of the experiment was sandy loam in texture with $\mathrm{pH} 6.6$, total $\mathrm{N} 0.08 \%$, available P $7.00 \mu \mathrm{g} / \mathrm{g}$, exchangeable K $0.07 \mathrm{me} / 100 \mathrm{~g}$ soil, and available $\mathrm{S} 7.5$ $\mu \mathrm{g} / \mathrm{g}$, respectively (Table 1). Each pot received $6.67 \mathrm{~kg}$ soil (weight in dry basis) with an opening at the bottom for collecting leachates. The upper surface area of the pot was $380 \mathrm{~cm}^{2}$. boro rice cv BRRI dhan 29 was used as the test crop. One hill (3 seedlings) was planted in each pot. The treatments were $\mathrm{T}_{0}$ (control), $\mathrm{T}_{1}$ $\left(\mathrm{N}_{120}, \mathrm{P}_{25}, \mathrm{~K}_{60}, \mathrm{~S}_{20} \mathrm{~kg} / \mathrm{ha}\right.$, recommended dose of fertilizer (BARC, 2005)), $\mathrm{T}_{2}$ $\left(\mathrm{N}_{180}, \mathrm{P}_{37.50}, \mathrm{~K}_{90}, \mathrm{~S}_{30} \mathrm{~kg} / \mathrm{ha}, 150 \%\right.$ of $\left.\mathrm{T}_{1}\right), \mathrm{T}_{3}\left(75 \% \mathrm{~N}\right.$ of $\mathrm{T}_{1}$ from chemical fertilizer and $25 \% \mathrm{~N}$ from cowdung $2.5 \mathrm{t} / \mathrm{ha}$ and PKS of recommended dose from chemical fertilizer on the basis of PKS content in cowdung), $\mathrm{T}_{4}$ (as $\mathrm{T}_{1}$ but $\mathrm{N}_{109} \mathrm{~kg} / \mathrm{ha}$ from USG) and $\mathrm{T}_{5}$ (as $\mathrm{T}_{1}$ but $\mathrm{N}$ applied as foliar spray). $\mathrm{N}, \mathrm{P}, \mathrm{K}$, and $\mathrm{S}$ content in cowdung was $1.2,1,1.6$, and $0.13 \%$, respectively. Fertilizers were applied as per treatments. All the fertilizers except urea were applied as basal to the individual pots and mixed well. The first split of urea was applied as top dressing after 10 days of transplanting boro rice followed by the second and third splits at 35 and 58 days after transplanting. In case of the treatment $\mathrm{T}_{4}$, one piece $(0.90 \mathrm{~g})$ of USG was placed in each pot after 10 days of transplanting. In that case, doses of $\mathrm{N}$ was $109 \mathrm{~kg} / \mathrm{ha}$. In case of foliar application of urea $\left(\mathrm{T}_{5}\right)$, onethird urea was applied after 10 days of transplanting and rest portion was sprayed at the rate of $3 \%$ in 6 equal splits at 10 days interval after the first application. Leachates from individual pots were collected in every day which were preserved in refrigerator and all leachates were mixed together at 15 days intervals and analyzed for $\mathrm{N}$ by rapid test method using soil testing kit, $\mathrm{P}$ by Olsen method (Olsen et al., 1954), K by flame photometer (Black, 1965) and S by turbidimetric method (Page et al., 1989). But in case of initial soil analysis, total $\mathrm{N}$ was 
determined by micro-Kjeldhal method (Page et al., 1989), texture by hydrometer method (Black, 1965) and $\mathrm{pH}$ by glass electrode $\mathrm{pH}$ meter method (Black, 1965).

Table 1. Physical and chemical characteristics of the initial soils.

\begin{tabular}{lc}
\multicolumn{1}{c|}{ Characteristics } & Results \\
\hline A. Physical characteristics & \\
$\%$ Sand & 46.8 \\
$\%$ Silt & 48.0 \\
$\%$ Clay & 5.2 \\
Soil texture & Sandy loam \\
B. Chemical characteristics & \\
Soil pH & 6.6 \\
Total N $(\%)$ & 0.08 \\
Available $\mathrm{P}(\mu \mathrm{g} / \mathrm{g})$ & 7.00 \\
Exchangeable K $(\mathrm{me} / 100 \mathrm{~g}$ soil $)$ & 0.07 \\
Available $\mathrm{S}(\mu \mathrm{g} / \mathrm{g})$ & 7.50 \\
\hline
\end{tabular}

\section{Results and Discussion}

Results presented in Table 2 indicated the leaching loss of N, P, and $\mathrm{K}$ in sandy loam soil due to different rates of fertilizer treatments under CSW condition over time. It was revealed that the leaching loss of nutrients from the fertilized pots was significantly higher over control irrespective of type of nutrients and study period. The maximum leaching loss of nutrients was recorded due to application of fertilizers at higher rates. The loss of $\mathrm{N}$ was maximum when urea was applied at higher rates $\left(\mathrm{T}_{2}\right)$ followed by urea application at recommended dose either as splits or foliar sprays. Application of $\mathrm{N}$ in the form of USG $\left(\mathrm{T}_{4}\right)$ reduced the leaching loss, while the minimum loss was recorded with integrated fertilization $\left(\mathrm{T}_{3}\right)$. Almost similar trend of leaching loss was observed in case of $\mathrm{P}$ and $\mathrm{K}$ also. The leaching loss of $\mathrm{NH}_{4}-\mathrm{N}, \mathrm{NO}_{3}-\mathrm{N}, \mathrm{P}$, and $\mathrm{K}$ in sandy loam soil due to different treatments under CSW condition at $1^{\text {st }} 15$ (1-15) days after transplanting (DAT) ranged from 1.50 to $5.50,0.50$ to $1.50,0.070$ to 0.225 , and 2.33 to $2.80 \mathrm{mg} / \mathrm{kg}$ soil, respectively, which was equivalent to 3.88 to $14.21,1.29$ to $3.88,0.183$ to 0.584 , and 6.02 to $7.24 \mathrm{~kg} / \mathrm{ha}$.

A significant variation in the leaching loss of $\mathrm{N}, \mathrm{P}$, and $\mathrm{K}$ due to different treatments in sandy loam soil under CSW condition was noted at $2^{\text {nd }} 15(16-30)$ DAT but the rate of losses was less as compared to $1^{\text {st }} 15$ days (Table 2). The leaching loss of $\mathrm{NH}_{4}-\mathrm{N}, \mathrm{NO}_{3}-\mathrm{N}, \mathrm{P}$ and $\mathrm{K}$ at $2^{\text {nd }} 15$ (16-30) DAT due to different treatments ranged from 1.40 to $5.20,0.40$ to $1.40,0.031$ to 0.154 , and 2.53 to $2.82 \mathrm{mg} / \mathrm{kg}$ soil, respectively, which was equivalent to 3.18 to $11.82,0.91$ to $3.18,0.071$ to 0.351 , and 5.75 to $6.36 \mathrm{~kg} / \mathrm{ha}$, respectively. During this period, the maximum leaching loss of nutrients was also observed in pots where higher rates of fertilizers were applied $\left(T_{2}\right)$. Integrated fertilization $\left(T_{3}\right)$ practice exhibited the 
Table 2. Leaching loss of $\mathrm{N}, \mathrm{P}$, and $\mathrm{K}$ due to different treatments from sandy loam soil under CSW condition over time at $\mathbf{1}^{\text {st }} \mathbf{1 5}$ (1-15) and $2^{\text {nd }} 15$ (16-30) days after transplanting (DAT).

\begin{tabular}{|c|c|c|c|c|c|c|c|c|c|}
\hline \multirow{2}{*}{ 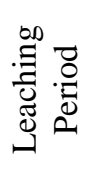 } & \multirow{2}{*}{ Treatments } & \multicolumn{2}{|c|}{ Available $\mathrm{NH}_{4}-\mathrm{N}$} & \multicolumn{2}{|c|}{ Available $\mathrm{NO}_{3}-\mathrm{N}$} & \multicolumn{2}{|c|}{ Available P } & \multicolumn{2}{|c|}{ Available K } \\
\hline & & $\mathrm{mg} / \mathrm{kg}$ & $\begin{array}{c}\text { Total loss } \\
\text { (kg/ha) }\end{array}$ & $\mathrm{mg} / \mathrm{kg}$ & $\begin{array}{c}\text { Total loss } \\
\text { (kg/ha) }\end{array}$ & $\mathrm{mg} / \mathrm{kg}$ & $\begin{array}{c}\text { Total loss } \\
(\mathrm{kg} / \mathrm{ha})\end{array}$ & $\mathrm{mg} / \mathrm{kg}$ & $\begin{array}{c}\text { Total loss } \\
\text { (kg/ha) }\end{array}$ \\
\hline \multirow{7}{*}{ 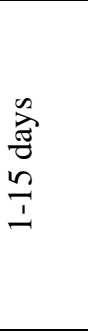 } & $\mathrm{T}_{0}$ & $1.50 \mathrm{~d}$ & $3.88 \mathrm{e}$ & $0.50 \mathrm{e}$ & $1.29 \mathrm{e}$ & $0.070 \mathrm{~d}$ & $0.183 \mathrm{e}$ & 2.33 & 6.02 \\
\hline & $\mathrm{T}_{1}$ & $4.50 \mathrm{~b}$ & $11.63 b$ & $1.10 \mathrm{~b}$ & $2.84 \mathrm{~b}$ & $0.155 \mathrm{~b}$ & $0.401 \mathrm{~b}$ & 2.66 & 6.87 \\
\hline & $\mathrm{T}_{2}$ & $5.50 \mathrm{a}$ & $14.21 \mathrm{a}$ & $1.50 \mathrm{a}$ & $3.88 \mathrm{a}$ & $0.225 \mathrm{a}$ & $0.584 \mathrm{a}$ & 2.80 & 7.24 \\
\hline & $\mathrm{T}_{3}$ & $3.50 \mathrm{c}$ & $9.05 \mathrm{~d}$ & $0.80 \mathrm{~d}$ & $2.07 \mathrm{~d}$ & $0.101 \mathrm{c}$ & $0.263 \mathrm{~d}$ & 2.46 & 6.36 \\
\hline & $\mathrm{T}_{4}$ & $4.00 \mathrm{bc}$ & $10.34 \mathrm{c}$ & $1.00 \mathrm{c}$ & $2.58 \mathrm{c}$ & $0.120 \mathrm{bc}$ & $0.310 \mathrm{bc}$ & 2.53 & 6.53 \\
\hline & $\mathrm{T}_{5}$ & $4.50 \mathrm{~b}$ & $11.63 \mathrm{~b}$ & $1.10 \mathrm{~b}$ & $2.84 \mathrm{~b}$ & $0.146 \mathrm{~b}$ & $0.380 \mathrm{c}$ & 2.60 & 6.72 \\
\hline & $\mathrm{SE}( \pm)$ & 0.554 & 1.430 & 0.137 & 0.354 & 0.021 & 0.056 & NS & NS \\
\hline \multirow{7}{*}{ 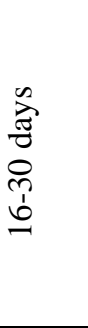 } & $\mathrm{T}_{0}$ & $1.40 \mathrm{~d}$ & $3.18 \mathrm{~d}$ & $0.40 \mathrm{~d}$ & $0.91 \mathrm{~d}$ & $0.031 \mathrm{c}$ & $0.071 \mathrm{c}$ & 2.53 & 5.75 \\
\hline & $\mathrm{T}_{1}$ & $4.20 \mathrm{~b}$ & $9.54 \mathrm{~b}$ & $1.00 \mathrm{~b}$ & $2.27 \mathrm{~b}$ & $0.101 \mathrm{ab}$ & $0.231 \mathrm{ab}$ & 2.66 & 6.04 \\
\hline & $\mathrm{T}_{2}$ & $5.20 \mathrm{a}$ & $11.82 \mathrm{a}$ & $1.40 \mathrm{a}$ & $3.18 \mathrm{a}$ & $0.154 \mathrm{a}$ & $0.351 \mathrm{a}$ & 2.82 & 6.36 \\
\hline & $\mathrm{T}_{3}$ & $3.20 \mathrm{c}$ & $7.27 \mathrm{c}$ & $0.75 \mathrm{c}$ & $1.70 \mathrm{c}$ & $0.055 \mathrm{bc}$ & $0.126 \mathrm{bc}$ & 2.60 & 5.91 \\
\hline & $\mathrm{T}_{4}$ & $3.40 \mathrm{c}$ & $7.73 \mathrm{c}$ & $0.90 \mathrm{c}$ & $2.05 \mathrm{c}$ & $0.063 \mathrm{bc}$ & $0.143 \mathrm{bc}$ & 2.62 & 5.95 \\
\hline & $\mathrm{T}_{5}$ & $3.70 \mathrm{c}$ & $8.41 \mathrm{c}$ & $0.70 \mathrm{c}$ & $1.59 \mathrm{c}$ & $0.074 \mathrm{bc}$ & $0.170 \mathrm{bc}$ & 2.60 & 5.91 \\
\hline & $\mathrm{SE}( \pm)$ & 0.510 & 1.169 & 0.137 & 0.310 & 0.018 & 0.040 & NS & NS \\
\hline
\end{tabular}

Figure(s) in a column having common letter(s) do not differ significantly at $5 \%$ level of significance.

$\mathrm{T}_{0}=$ control, $\mathrm{T}_{1}=\mathrm{N}_{120} \mathrm{P}_{25} \mathrm{~K}_{60}$ and $\mathrm{S}_{20} \mathrm{~kg} / \mathrm{ha}, \mathrm{T}_{2}=\mathrm{N}_{180} \mathrm{P}_{37.50} \mathrm{~K}_{90}$, and $\mathrm{S}_{30} \mathrm{~kg} / \mathrm{ha}, \mathrm{T}_{3}=75 \% \mathrm{~N}$ of $\mathrm{T}_{1}$ from chemical fertilizer and $25 \%$

$\mathrm{N}$ from cow dung $2.5 \mathrm{t} / \mathrm{ha}$ and PKS of recommended dose from chemical fertilizer on the basis of PKS content in cowdung, $\mathrm{T}_{4}=$ As

$\mathrm{T}_{1}$ but $\mathrm{N}_{109} \mathrm{~kg} / \mathrm{ha}$ from USG and $\mathrm{T}_{5}=$ As $\mathrm{T}_{1}$ but $\mathrm{N}$ applied as foliar spray. 
Table 3. Leaching loss of $\mathrm{N}, \mathrm{P}$, and $\mathrm{K}$ as influenced by different fertilizer treatments from sandy loam soil under CSW condition at $3^{\text {rd }} 15(31-45)$ and $4^{\text {th }} 15(46-60)$ days after transplanting (DAT).

\begin{tabular}{|c|c|c|c|c|c|c|c|c|c|}
\hline \multirow{2}{*}{ 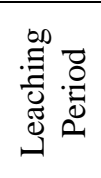 } & \multirow{2}{*}{ Treatments } & \multicolumn{2}{|c|}{ Available $\mathrm{NH}_{4}-\mathrm{N}$} & \multicolumn{2}{|c|}{ Available $\mathrm{NO}_{3}-\mathrm{N}$} & \multicolumn{2}{|c|}{ Available P } & \multicolumn{2}{|c|}{ Available K } \\
\hline & & $\mathrm{mg} / \mathrm{kg}$ & $\begin{array}{c}\text { Total loss } \\
\text { (kg/ha) }\end{array}$ & $\mathrm{mg} / \mathrm{kg}$ & $\begin{array}{c}\text { Total loss } \\
\text { (kg/ha) }\end{array}$ & $\mathrm{mg} / \mathrm{kg}$ & $\begin{array}{c}\text { Total loss } \\
\text { (kg/ha) }\end{array}$ & $\mathrm{mg} / \mathrm{kg}$ & $\begin{array}{c}\text { Total loss } \\
\text { (kg/ha) }\end{array}$ \\
\hline \multirow{7}{*}{$\begin{array}{l}\text { 胥 } \\
\text { in } \\
\frac{i}{1} \\
\frac{1}{n}\end{array}$} & $\mathrm{~T}_{0}$ & $1.30 \mathrm{c}$ & $2.56 \mathrm{~d}$ & $0.35 \mathrm{~d}$ & $0.69 \mathrm{f}$ & $0.011 \mathrm{c}$ & $0.020 \mathrm{c}$ & $2.50 \mathrm{c}$ & $4.93 \mathrm{~b}$ \\
\hline & $\mathrm{T}_{1}$ & $4.25 \mathrm{a}$ & $8.38 \mathrm{~b}$ & $1.10 \mathrm{~b}$ & $2.17 \mathrm{~b}$ & $0.062 \mathrm{~b}$ & $0.124 b$ & $2.90 \mathrm{a}$ & $5.75 \mathrm{a}$ \\
\hline & $\mathrm{T}_{2}$ & $5.30 \mathrm{a}$ & $10.45 \mathrm{a}$ & $1.45 \mathrm{a}$ & $2.86 \mathrm{a}$ & $0.103 \mathrm{a}$ & $0.204 \mathrm{a}$ & $2.93 \mathrm{a}$ & $5.77 \mathrm{a}$ \\
\hline & $\mathrm{T}_{3}$ & $3.60 \mathrm{ab}$ & $7.09 \mathrm{bc}$ & $0.85 \mathrm{bc}$ & $1.68 \mathrm{c}$ & $0.012 \mathrm{c}$ & $0.024 \mathrm{c}$ & $2.86 \mathrm{~b}$ & $5.64 \mathrm{a}$ \\
\hline & $\mathrm{T}_{4}$ & $3.45 a b$ & $6.80 \mathrm{bc}$ & $0.80 \mathrm{c}$ & $1.58 \mathrm{~d}$ & $0.012 \mathrm{c}$ & $0.024 \mathrm{c}$ & $2.83 \mathrm{bc}$ & $5.58 \mathrm{a}$ \\
\hline & $\mathrm{T}_{5}$ & $3.20 \mathrm{~b}$ & $6.31 \mathrm{c}$ & $0.70 \mathrm{c}$ & $1.38 \mathrm{e}$ & $0.016 \mathrm{c}$ & $0.032 \mathrm{c}$ & $2.86 \mathrm{~b}$ & $5.64 \mathrm{a}$ \\
\hline & $\mathrm{SE}( \pm)$ & 0.540 & 1.064 & 0.152 & 0.300 & 0.016 & 0.031 & 0.064 & 0.128 \\
\hline \multirow{7}{*}{ 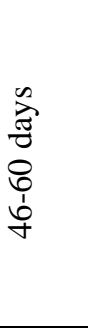 } & $\mathrm{T}_{0}$ & $1.25 \mathrm{c}$ & $2.67 \mathrm{~d}$ & $0.30 \mathrm{c}$ & $0.64 \mathrm{f}$ & $0.032 \mathrm{c}$ & $0.070 \mathrm{~d}$ & $2.60 \mathrm{c}$ & $5.55 \mathrm{~b}$ \\
\hline & $\mathrm{T}_{1}$ & $4.00 \mathrm{ab}$ & $8.54 \mathrm{~b}$ & $1.00 \mathrm{ab}$ & $2.13 \mathrm{~b}$ & $0.104 \mathrm{a}$ & $0.222 \mathrm{a}$ & $2.96 \mathrm{~b}$ & $6.32 \mathrm{a}$ \\
\hline & $\mathrm{T}_{2}$ & $5.10 \mathrm{a}$ & $10.89 \mathrm{a}$ & $1.30 \mathrm{a}$ & $2.77 \mathrm{a}$ & $0.106 \mathrm{a}$ & $0.228 \mathrm{a}$ & $3.06 \mathrm{a}$ & $6.53 \mathrm{a}$ \\
\hline & $\mathrm{T}_{3}$ & $3.35 \mathrm{~b}$ & $7.15 \mathrm{bc}$ & $0.70 \mathrm{~b}$ & $1.49 \mathrm{~d}$ & $0.043 \mathrm{bc}$ & $0.094 \mathrm{c}$ & $2.90 \mathrm{~b}$ & $6.19 \mathrm{a}$ \\
\hline & $\mathrm{T}_{4}$ & $3.50 \mathrm{~b}$ & $7.47 \mathrm{bc}$ & $0.75 \mathrm{~b}$ & $1.60 \mathrm{c}$ & $0.049 \mathrm{bc}$ & $0.105 \mathrm{bc}$ & $2.92 \mathrm{~b}$ & $6.23 \mathrm{a}$ \\
\hline & $\mathrm{T}_{5}$ & $3.00 \mathrm{~b}$ & $6.40 \mathrm{c}$ & $0.65 \mathrm{~b}$ & $1.39 \mathrm{e}$ & $0.052 \mathrm{~b}$ & $0.111 \mathrm{~b}$ & $2.95 \mathrm{~b}$ & $6.30 \mathrm{a}$ \\
\hline & $\mathrm{SE}( \pm)$ & 0.518 & 1.106 & 0.138 & 0.294 & 0.013 & 0.028 & 0.063 & 0.136 \\
\hline
\end{tabular}

Figure(s) in a column having common letter(s) do not differ significantly at $5 \%$ level of significance.

$\mathrm{T}_{0}=$ control, $\mathrm{T}_{1}=\mathrm{N}_{120} \mathrm{P}_{25} \mathrm{~K}_{60}$, and $\mathrm{S}_{20} \mathrm{~kg} / \mathrm{ha}, \mathrm{T}_{2}=\mathrm{N}_{180} \mathrm{P}_{37.50} \mathrm{~K}_{90}$, and $\mathrm{S}_{30} \mathrm{~kg} / \mathrm{ha}, \mathrm{T}_{3}=75 \% \mathrm{~N}$ of $\mathrm{T}_{1}$ from chemical fertilizer and $25 \%$

$\mathrm{N}$ from cowdung $2.5 \mathrm{t} / \mathrm{ha}$ and PKS of recommended dose from chemical fertilizer on the basis of PKS content in cowdung, $\mathrm{T}_{4}=\mathrm{As}$

$\mathrm{T}_{1}$ but $\mathrm{N}_{109} \mathrm{~kg} / \mathrm{ha}$ from USG and $\mathrm{T}_{5}=\mathrm{As} \mathrm{T}_{1}$ but $\mathrm{N}$ applied as foliar spray. 
Table 4. Leaching loss of $\mathrm{N}, \mathrm{P}$, and $\mathrm{K}$ as influenced by different fertilizer treatments from sandy loam soil under CSW condition at $5^{\text {th }} 15(61-75)$ and $6^{\text {th }} 15(76-90)$ days after transplanting (DAT).

\begin{tabular}{|c|c|c|c|c|c|c|c|c|c|}
\hline \multirow{2}{*}{ 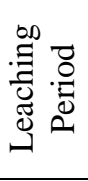 } & \multirow{2}{*}{ Treatments } & \multicolumn{2}{|c|}{ Available $\mathrm{NH}_{4}-\mathrm{N}$} & \multicolumn{2}{|c|}{ Available $\mathrm{NO}_{3}-\mathrm{N}$} & \multicolumn{2}{|c|}{ Available P } & \multicolumn{2}{|c|}{ Available $\mathrm{K}$} \\
\hline & & $\mathrm{mg} / \mathrm{kg}$ & $\begin{array}{l}\text { Total loss } \\
(\mathrm{kg} / \mathrm{ha})\end{array}$ & $\mathrm{mg} / \mathrm{kg}$ & $\begin{array}{c}\text { Total loss } \\
\text { (kg/ha) }\end{array}$ & $\mathrm{mg} / \mathrm{kg}$ & $\begin{array}{c}\text { Total loss } \\
\text { (kg/ha) }\end{array}$ & $\mathrm{mg} / \mathrm{kg}$ & $\begin{array}{c}\text { Total loss } \\
\text { (kg/ha) }\end{array}$ \\
\hline \multirow{7}{*}{$\begin{array}{l}\sum_{\overrightarrow{7}}^{\infty} \\
\frac{n}{2} \\
\frac{1}{6}\end{array}$} & $\mathrm{~T}_{0}$ & $1.20 \mathrm{e}$ & $2.65 \mathrm{~d}$ & $0.25 \mathrm{~d}$ & $0.60 \mathrm{~d}$ & $0.035 \mathrm{~d}$ & $0.087 \mathrm{c}$ & $2.60 \mathrm{c}$ & $6.27 \mathrm{c}$ \\
\hline & $\mathrm{T}_{1}$ & $4.10 \mathrm{~b}$ & $9.88 \mathrm{~b}$ & $1.10 \mathrm{~b}$ & $2.65 \mathrm{~b}$ & $0.088 \mathrm{~b}$ & $0.214 \mathrm{a}$ & $3.05 \mathrm{ab}$ & $7.35 \mathrm{a}$ \\
\hline & $\mathrm{T}_{2}$ & $5.15 \mathrm{a}$ & $12.41 \mathrm{a}$ & $1.35 \mathrm{a}$ & $3.25 \mathrm{a}$ & $0.101 \mathrm{a}$ & $0.245 \mathrm{a}$ & $3.20 \mathrm{a}$ & $7.71 \mathrm{a}$ \\
\hline & $\mathrm{T}_{3}$ & $3.40 \mathrm{c}$ & $8.19 \mathrm{c}$ & $0.75 \mathrm{c}$ & $1.81 \mathrm{bc}$ & $0.059 \mathrm{c}$ & $0.143 \mathrm{~b}$ & $2.94 \mathrm{~b}$ & $7.09 \mathrm{~b}$ \\
\hline & $\mathrm{T}_{4}$ & $3.30 \mathrm{c}$ & $7.95 \mathrm{c}$ & $0.60 \mathrm{c}$ & $1.45 \mathrm{c}$ & $0.061 \mathrm{c}$ & $0.148 \mathrm{~b}$ & $2.82 \mathrm{~b}$ & $6.80 \mathrm{bc}$ \\
\hline & $\mathrm{T}_{5}$ & $2.95 \mathrm{~d}$ & $7.11 \mathrm{~cd}$ & $0.65 \mathrm{c}$ & $1.57 \mathrm{c}$ & $0.061 \mathrm{c}$ & $0.148 \mathrm{~b}$ & $2.97 \mathrm{~b}$ & $7.16 \mathrm{~b}$ \\
\hline & $\mathrm{SE}( \pm)$ & $\mathbf{0 . 5 3 5}$ & 1.321 & 0.159 & 0.383 & 0.009 & 0.023 & 0.084 & 0.201 \\
\hline \multirow{7}{*}{ 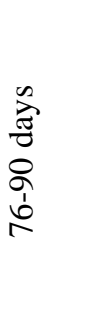 } & $\mathrm{T}_{0}$ & $1.00 \mathrm{c}$ & $2.63 \mathrm{c}$ & $0.20 \mathrm{~d}$ & $0.53 \mathrm{~d}$ & $0.077 \mathrm{c}$ & $0.203 \mathrm{c}$ & $2.55 \mathrm{c}$ & $6.70 \mathrm{c}$ \\
\hline & $\mathrm{T}_{1}$ & $3.70 \mathrm{ab}$ & $9.72 a b$ & $0.90 \mathrm{ab}$ & $2.36 \mathrm{ab}$ & $0.121 \mathrm{a}$ & $0.318 \mathrm{a}$ & $3.10 \mathrm{ab}$ & $8.14 \mathrm{ab}$ \\
\hline & $\mathrm{T}_{2}$ & $4.70 \mathrm{a}$ & $12.34 \mathrm{a}$ & $1.20 \mathrm{a}$ & $3.15 \mathrm{a}$ & $0.124 \mathrm{a}$ & $0.335 \mathrm{a}$ & $3.22 \mathrm{a}$ & $8.40 \mathrm{a}$ \\
\hline & $\mathrm{T}_{3}$ & $3.00 \mathrm{~b}$ & $7.88 \mathrm{~b}$ & $0.65 \mathrm{bc}$ & $1.71 \mathrm{bc}$ & $0.093 \mathrm{~b}$ & $0.247 \mathrm{~b}$ & $2.96 \mathrm{~b}$ & $7.77 \mathrm{~b}$ \\
\hline & $\mathrm{T}_{4}$ & $3.00 \mathrm{~b}$ & $7.88 \mathrm{~b}$ & $0.50 \mathrm{~cd}$ & $1.31 \mathrm{~cd}$ & $0.090 \mathrm{~b}$ & $0.236 \mathrm{~b}$ & $2.90 \mathrm{~b}$ & $7.62 \mathrm{~b}$ \\
\hline & $\mathrm{T}_{5}$ & $2.60 \mathrm{~b}$ & $6.83 \mathrm{~b}$ & $0.45 \mathrm{~cd}$ & $1.18 \mathrm{~cd}$ & $0.098 \mathrm{~b}$ & $0.258 \mathrm{~b}$ & $3.00 \mathrm{ab}$ & $7.88 \mathrm{ab}$ \\
\hline & $\mathrm{SE}( \pm)$ & 0.4143 & 1.0880 & 0.0962 & 0.2526 & 0.0028 & 0.0075 & 0.0628 & 0.1649 \\
\hline
\end{tabular}

Figure(s) in a column having common letter(s) do not differ significantly at $5 \%$ level of significance.

$\mathrm{T}_{0}=$ control, $\mathrm{T}_{1}=\mathrm{N}_{120} \mathrm{P}_{25} \mathrm{~K}_{60}$ and $\mathrm{S}_{20} \mathrm{~kg} / \mathrm{ha}, \mathrm{T}_{2}=\mathrm{N}_{180} \mathrm{P}_{37.50} \mathrm{~K}_{90}$, and $\mathrm{S}_{30} \mathrm{~kg} / \mathrm{ha}, \mathrm{T}_{3}=75 \% \mathrm{~N}$ of $\mathrm{T}_{1}$ from chemical fertilizer and $25 \%$

$\mathrm{N}$ from cowdung $2.5 \mathrm{t} / \mathrm{ha}$ and PKS of recommended dose from chemical fertilizer on the basis of PKS content in cowdung, $\mathrm{T}_{4}=\mathrm{As}$

$\mathrm{T}_{1}$ but $\mathrm{N}_{109} \mathrm{~kg} / \mathrm{ha}$ from USG and $\mathrm{T}_{5}=$ As $\mathrm{T}_{1}$ but $\mathrm{N}$ applied as foliar spray. 
minimum loss. Similarly, at $3^{\text {rd }} 15$ DAT (31-45), the leaching loss of $\mathrm{NH}_{4}-\mathrm{N}$, $\mathrm{NO}_{3}-\mathrm{N}, \mathrm{P}$, and $\mathrm{K}$ varied from 1.30 to $5.30,0.35$ to $1.45,0.011$ to 0.103 , and 2.50 to $2.93 \mathrm{mg} / \mathrm{kg}$ soil, respectively, which was equivalent to 2.56 to $10.45,0.69$ to $2.86,0.020$ to 0.204 , and 4.93 to $5.77 \mathrm{~kg} / \mathrm{ha}$ (Table 3 ). The corresponding values of leaching loss of $\mathrm{NH}_{4}-\mathrm{N}, \mathrm{NO}_{3}-\mathrm{N}, \mathrm{P}$ and $\mathrm{K}$ at $4^{\text {th }} 15$ DAT (46-60) ranged from 1.25 to $5.10,0.30$ to $1.30,0.032$ to 0.106 , and 2.60 to $3.06 \mathrm{mg} / \mathrm{kg}$ soil, respectively, which was equivalent to 2.67 to $10.89,0.64$ to $2.77,0.070$ to 0.228 , and 5.55 to $6.53 \mathrm{~kg} / \mathrm{ha}$ (Table 3). The leaching loss of $\mathrm{NH}_{4}-\mathrm{N}, \mathrm{NO}_{3}-\mathrm{N}, \mathrm{P}$, and $\mathrm{K}$ in sandy loam soil due to different treatments under CSW condition at $5^{\text {th }}$ DAT (61-75) ranged from 1.20 to $5.15,0.25$ to $1.35,0.035$ to 0.101 , and 2.60 to 3.20 $\mathrm{mg} / \mathrm{kg}$ soil, respectively (Table 4). Leaching loss of N, P, and $\mathrm{K}$ at $6^{\text {th }}$ DAT (76 to 90 ), varied significantly due to different treatments in sandy loam soil under CSW condition (Table 4). The leaching loss of $\mathrm{NH}_{4}-\mathrm{N}, \mathrm{NO}_{3}-\mathrm{N}, \mathrm{P}$, and $\mathrm{K}$ during this period ranged from 1.00 to $4.70,0.20$ to $1.20,0.077$ to 0.124 , and 2.55 to $3.22 \mathrm{mg} / \mathrm{kg}$ soil, respectively, which was equivalent to 2.63 to $12.34,0.53$ to $3.15,0.203$ to 0.335 , and 6.70 to $8.40 \mathrm{~kg} / \mathrm{ha}$ (Table 4 ).

The loss of $\mathrm{NH}_{4}-\mathrm{N}$ for the treatment $\mathrm{T}_{1}$ was $4.5 \mathrm{mg} / \mathrm{kg}$ at 15 DAT, which was reduced by $6.7 \%$ at 30 DAT and then remained almost static up to 75 DAT but sharply reduced by $17.8 \%$ at 90 DAT showing a loss of $3.70 \mathrm{mg} / \mathrm{kg}$. Similarly, at 15 DAT loss of $\mathrm{NO}_{3}-\mathrm{N}$ was $1.10 \mathrm{mg} / \mathrm{kg}$ which reduced by $9.1 \%$ at 30 DAT then at 45 DAT amount of loss increased again as like as at 15 DAT. Intensity of $\mathrm{N}$ loss was increased after the urea top dress. The loss was highly reduced by $18.2 \%$ at 90 DAT. Almost similar trend was found in other treatments except USG treatment. In that case, loss was very high at 15 DAT in comparison to other growing periods. P loss was low at 45 DAT in comparison to 60, 75, and 90 DAT. It might be due to more requirements of $\mathrm{P}$ at $45 \mathrm{DAT}$ for reproduction of plant. $\mathrm{K}$ loss gradually increased with the growing period.

Table 5. Total leaching loss of NPKS from sandy loam soil under CSW condition.

\begin{tabular}{c|ccc|cc|c}
\hline \multirow{2}{*}{ Treatment } & \multicolumn{3}{|c|}{$\mathrm{N} \mathrm{(kg/ha)}$} & $\mathrm{P}$ & $\mathrm{K}$ \\
\cline { 2 - 6 } & $\mathrm{NH}_{4}-\mathrm{N}$ & $\mathrm{NO}_{3}-\mathrm{N}$ & Total & \multicolumn{2}{|c}{$(\mathrm{kg} / \mathrm{ha})$} \\
\hline $\mathrm{T}_{0}$ & 17.57 & 4.66 & 22.23 & 0.63 & 35.22 \\
$\mathrm{~T}_{1}$ & 57.69 & 14.42 & 72.11 & 1.51 & 40.47 \\
$\mathrm{~T}_{2}$ & 72.12 & 19.09 & 91.21 & 1.95 & 42.01 \\
$\mathrm{~T}_{3}$ & 46.63 & 10.46 & 57.09 & 0.90 & 38.96 \\
$\mathrm{~T}_{4}$ & 48.17 & 10.57 & 58.74 & 0.97 & 38.71 \\
$\mathrm{~T}_{5}$ & 46.69 & 9.95 & 56.64 & 1.10 & 39.61 \\
\hline
\end{tabular}

The total leaching loss of $\mathrm{NH}_{4}-\mathrm{N}, \mathrm{NO}_{3}-\mathrm{N}$, $\mathrm{P}$, and $\mathrm{K}$ during the growing period (90 days) of boro rice due to different treatments under CSW condition ranged from 17.57 to $72.12,4.66$ to $19.09,0.63$ to 1.95 , and 35.22 to 42.01 
$\mathrm{kg} / \mathrm{ha}$, respectively (Table 5). The total $\mathrm{N}$ loss during the period ranged from 22.23 to $91.21 \mathrm{~kg} / \mathrm{ha}$. About 41.6, 38.3, 29.1, 33.5, and $28.7 \%$ of applied $\mathrm{N}$ was lost through leaching for the treatment $\mathrm{T}_{1}, \mathrm{~T}_{2}, \mathrm{~T}_{3}, \mathrm{~T}_{4}$, and $\mathrm{T}_{5}$, respectively. Leaching loss of $\mathrm{N}$ in the form of $\mathrm{NH}_{4}-\mathrm{N} 33.4 \%$ while $8.1 \%$ applied $\mathrm{N}$ was lost as $\mathrm{NO}_{3}-\mathrm{N}$ for the treatment $\mathrm{T}_{1}$. In the other treatments, almost similar trend was found (Table 5). Leaching loss of $\mathrm{P}$ for the treatment $\mathrm{T}_{1}, \mathrm{~T}_{2}, \mathrm{~T}_{3}, \mathrm{~T}_{4}$, and $\mathrm{T}_{5}$ was $3.52,3.52,1.08,1.36$, and $1.88 \%$, respectively. Another nutrient $\mathrm{K}$ was lost through leaching $\mathrm{T}_{0}=$ Control, $\mathrm{T}_{1}=\mathrm{N}_{120} \mathrm{P}_{25} \mathrm{~K}_{60} \mathrm{~S}_{20} \mathrm{~kg} / \mathrm{ha}, \mathrm{T}_{2}=\mathrm{N}_{180} \mathrm{P}_{37.5} \mathrm{~K}_{90} \mathrm{~S}_{30}$ $\mathrm{kg} / \mathrm{ha}, \mathrm{T}_{3}=75 \% \mathrm{~N}$ of $\mathrm{T}_{1}$ from chemical fertilizer and $25 \% \mathrm{~N}$ from cowdung 2.5 $\mathrm{t} / \mathrm{ha}$ and PKS of recommended dose from chemical fertilizer on the basis of PKS content in cowdung, $\mathrm{T}_{4}=$ As $\mathrm{T}_{1}$ but $\mathrm{N}_{109} \mathrm{~kg} / \mathrm{ha}$ from USG, $\mathrm{T}_{5}=\mathrm{As} \mathrm{T}_{1}$ but $\mathrm{N}$ foliar spray was $8.8,7.5,6.2,5.8$, and $7.3 \%$ for the treatment $\mathrm{T}_{1}, \mathrm{~T}_{2}, \mathrm{~T}_{3}, \mathrm{~T}_{4}$, and $\mathrm{T}_{5}$, respectively. Application of fertilizers at higher rate resulted more leaching loss of nutrients, while the minimum loss of nutrients was noted with the integrated fertilization. Although losses of nutrients in percentage of $T_{2}$ treatments were lower than that of $T_{1}$, total losses of nutrients were more in the treatment $T_{2}$. The slow release of nutrients from the organic manure might have resulted in low loss of nutrients for $\mathrm{T}_{3}$. Again, USG releases $\mathrm{N}$ slowly which contributed to low loss of $\mathrm{N}$. Losses of $\mathrm{P}$ was minimum in the USG treated pot, it might be due to more vegetative growth of plant and more nutrient uptake. The reason for low loss of $\mathrm{N}$ from the foliar spray treatment is quite obvious.

\section{Conclusion}

Leaching loss of essential plant nutrients like N, P, and K from the sandy soil of Old Brahmaputra Floodplain (AEZ-9) under Continuous Standing Water (CSW) condition for boro rice cultivation was quite significant. Application of chemical fertilizer at higher rates resulted in greater loss of nutrients. Integrated approach of fertilizer management could minimize such losses to a great extent. Application of $\mathrm{N}$ in the form of USG may be helpful in reducing the $\mathrm{N}$ loss appreciably.

\section{References}

BARC. 2005. Fertilizer Recommendation Guide-2005. Bangladesh Agricultural Research Council, Farmgate, Dhaka-1215.

Black, C. A. 1965. Methods of Soil Analysis. Part I \& II. American Soc. Agron. Inc. Pub. Madison, Wisconsin, USA.

Camberato, J., J. Brad and R. L. Nielsen. 2008. Nitrogen loss in wet and wetter fields. Corney News Network, Purdue University. URL: http://www.kingcorn.org/

de Oliveira, M. W., P. C. O. Trivelin, A. E. Boaretto, T. Muraoka and J. Mortatti. 2002. Leaching of nitrogen, potassium, calcium and magnesium in a sandy soil cultivated with sugarcane. J. Pesq. Agropec. Bras., Brasilia 37(6):861-868. 
Donova, G. and C. Casey, 1998. Soil fertility management in Sub-Saharan AfricaPhosphorus and nitrogen based manure and compost application. J. Agron. 94:128-135.

Fahmi A., B. Radjagukguk and B. H. Purwanto. 2012. The leaching of iron and loss of phosphate in acid Sulphate soil due to rice straw and phosphate fertilizer Application. J. Trop. Soils 17 (1): 19-24.

Olsen, S. R., C. V. Cole, F. S. Watanabe and L. A. Dean. 1954. Estimation of available phosphorus in soils by extraction with sodium bicarbonate. U. S. Dept. Agric. Circ. 939.

Page, A. L., R. H. Miller and D. R. Keeney. 1989. Methods of Soil Analysis. Part II. $2^{\text {nd }}$ ed. Amer. Soc. of Agron. Inc. Pub. Madison, Wisconsin, USA.

Pieri C. and R. Oliver. 1986. Assessment of K losses in tropical coropping systems of francophone Africa and Madagascar. Proceeding $13^{\text {th }}$ Congress International Potash Institure, Bern: 73-92.

Sharpley, A. N. 1997. Rainfall frequency and nitrogen and phosphorus runoff from soil amended with poultry litter. J. Environ. Qual. 26: 1127-1132.

Sahu S. K. and P. K. Samant. 2006. Nitrogen loss from rice soils in Orissa. Orissa Review-2006, Dept. of Soil Sci. and Agril. Chem. (O.U.A.T), Bhubaneswar.

Toor, G. S., L. M. Condron, B. J. Cade-Menun. H. J. Di, and K. C. Cameron. 2004. Preferential phosphorus leaching from an irrigated grassland soil. Fur. J. Soil Sci.

Vlek, P. L. G., B. H. Byrnes, and E. T. Craswell. 1980 Effect of urea placement on leaching losses of nitrogen from flooded rice soils. J. Plant Soil 54: 441-449.

Velu, V. and K.M. Ramanathan. 2001. Nitrogen balance in wetland rice ecosystem as influenced by soil type. J. Madras Agril. 87: 21-25.

Xing, G. X. and Z. L. Zhu, 2000. An assessment of N loss from agricultural fields to the environment in China. Nutrient Cycling in Agroecosystems 57: 67-73. 